\title{
FUNCIONAMIENTO FAMILIAR Y RE- SILIENCIA EN ESTUDIANTES DE QUINTO AÑO DE TEOLOGÍA DE LA UNIVERSIDAD PERUANA UNIÓN
}

\author{
Zinia K. Yupanqui Illacutipa \\ Lea L. Ingaroca Pizarro \\ Andy A. Carbajal Cerrinos \\ Facultad de Ciencias Humanas y Educación \\ Universidad Peruana Unión \\ zinia@hotmail.es/lita_15_5@hotmail.com \\ Fecha de recepción:Mayo 2012 \\ Fecha de aceptación y versión final: Julio 2012
}

\section{Resumen} La resiliencia es la capacidad que permite a la persona salir airosa de las amenazas y problemas a los cuales se enfrenta. En este estudio se intenta verificar si existe relación significativa entre el funcionamiento familiar y resiliencia en estudiantes de teología de quinto año de la Universidad Peruana Unión de Lima en el año 2011. El estudio es de tipo cuantitativo, descriptivo, correlacional y de diseño no experimental y transversal. En cuanto a los instrumentos utilizados fueron: la escala de resiliencia de Wagnild y Young y el cuestionario de funcionamiento familiar de McMaster elaborado por Atri y Zetune.

Los resultados manifiestan que el funcionamiento familiar se relaciona significativamente con el nivel de resiliencia de los estudiantes, ya que califican en un nivel alto de resiliencia y con más funcionalidad en la mayoría de las dimensiones del funcionamiento familiar. Adicionalmente se encontró que las dimensiones de involucramiento afectivo y resolución de problemas se relacionan significativamente con el nivel de resiliencia. En cambio, la dimensión patrones de conducta se relaciona moderadamente y la dimensión patrones de comunicación no se relaciona significativamente con la resiliencia.

Palabras clave: Funcionamiento familiar, resiliencia. 
"La edificación del carácter es la obra más importante que jamás haya sido confiada a los seres humanos y nunca antes ha sido su estudio diligente tan importante como ahora. Ninguna generación anterior fue llamada a hacer frente a problemas tan importantes; nunca antes se hallaron los jóvenes frente a peligros tan grandes como los que tienen que arrostrar hoy".

White Elena G. de. Conducción del Niño. p. 155

\section{Introducción}

Los seres humanos somos influenciados permanentemente, desde el momento de la concepción hasta el momento antes de morir; somos capaces de 8 afectar las vidas de quienes nos rodean así como ellos influyen en las nuestras. La familia, que es considerada el centro de la sociedad, el corazón de la iglesia, es el lugar donde sus miembros forman una sociedad básica con su propia cultura, su original proceso social, su nivel estratégico de comunicación, sus códigos específicos, ademanes singulares y pensamientos propios.

Es la familia la que provee, al futuro ciudadano, las herramientas necesarias para enfrentar la vida y, de manera específica, para confrontar los problemas y dificultades. Es en su funcionamiento familiar que ayuda a desarrollar, en sus miembros, la capacidad de sobresalir a los problemas y aprender de ellos, a esa capacidad la psicología le ha dado el nombre de resiliencia.

La familia juega un importante papel, principalmente los padres en la formación de la resiliencia en sus hijos. Para concluir, se puede decir que aspectos tales como la estructura de la familia de origen, así como muchas de las características de los padres influyen en los patrones de conducta y, por ende, en la formación de resiliencia de los individuos.

Con lo dicho hasta aquí, la familia se la puede ver como un sistema de gran influencia a nivel preventivo, ya que puede desarrollar actitudes y comportamientos para enfrentar los factores de riesgo, una fuente relevante que proporciona al individuo herramientas y recursos para enfrentar la adversidad. Algunas de las contribuciones que da la familia a la resiliencia del individuo incluyen como las relaciones de confianza y de cuidado; el desarrollo de un propósito positivo en las expectativas de la vida, soportar decisiones que se han tomado y proveer una familia con cohesión y apoyo. Se sugiere que el ambiente familiar es 
un factor importante para producir la resiliencia en los niños.

Es por ello que se plantea la siguiente pregunta iExiste relación significativa entre la resiliencia y el funcionamiento familiar en los alumnos de 5 to año de la Facultad de Teología de la Universidad Peruana Unión?

El objetivo de este estudio fue determinar si existe relación entre el funcionamiento familiar y resiliencia en los alumnos de 5to año de la Facultad de Teología de la Universidad Peruana Unión.

\section{Funcionamiento familiar}

\section{Definición de funciona- miento familiar}

La familia es el sistema de relaciones fundamentalmente afectivas, presente en todas las culturas, en el que el ser humano permanece largo tiempo, $y$ no un tiempo cualquiera de su vida, sino el formado por sus fases evolutivas cruciales (neonatal, infancia y adolescencia).

Se considera que la familia es un grupo o sistema compuesto por subsistemas que serían sus propios miembros. Tomando en cuenta el aspecto funcional cada uno de los miembros tiene diferentes funciones que varían en el tiempo y que depende de la edad, el sexo y la interacción con otros miembros de la familia. Es en este contexto que se puede entender las relaciones de la familia como una red de influencias recíprocas tendientes a lograr los objetivos comunes a todos ellos.

\section{Dimensiones del modelo McMaster de funciona- miento familiar}

El modelo McMaster de funcionamiento familiar identifica un gran número de dimensiones que han resultado ser de utilidad en la evaluación de las familias, además permite obtener una descripción clara de lo que es una familia normal por medio de seis dimensiones como son la resolución de problemas, comunicación, roles, involucramiento afectivo, respuestas afectivas y control de conducta.

Involucramiento afectivo funcional representa el grado en que la familia muestra interés y valora a cada miembro de la familia y denota la habilidad para responder con una amplia gama 
de emociones, así como la capacidad de proporcionar cuidados, afecto y bienestar.

El "involucramiento afectivo disfuncional" implica una sobrecarga en algún miembro de la familia que lo convierte en un receptor de los aspectos negativos de la familia, es decir el que recibe toda la tención de los demás.

Los "patrones de comunicación disfuncionales" están enfocados a una marcada dificultad en el intercambio de información en el área afectiva.

Los "patrones de comunicación funcionales" tiene que ver con los aspectos positivos de la comunicación, se refiere al incremento de la información verbal, principalmente en el área instrumental.

El factor "resolución de problemas en la familia" define cuándo una familia es capaz de ponerse de acuerdo y que tiene una buena organización familiar, por lo tanto, se infiere que puedan resolver los problemas instrumentales que se les presentan.

Los "patrones de control de conducta" se refieren a los patrones que adopta una familia para manejar el comportamiento, siendo estos patrones razonables con la posibilidad de negociación y cambio, dependiendo del contexto.

\section{Resiliencia}

\section{Concepto de resiliencia}

Etimológicamente el témino resiliencia proviene de la palabra latina "resilio" que se utiliza en la física y significa rebotar, volver. También expresa la cualidad de resistencia de los materiales a la presión, al doblarse y recobrar su forma original, es decir tiene la propiedad de resistir a las presiones externas y, a pesar de esas presiones, no deformarse.

En el área de psicología el término de resiliencia se ha empezado a utilizar en 1972 por los psicólogos Kobasa y Maddi quienes desarrollaron el concepto de resiliencia. Este concepto denota la capacidad de protección ante factores estresantes. En un contexto cotidiano indica la capacidad de las personas que, ante un hecho negativo, reaccionan favorablemente gracias a ciertas características de personalidad, las cuales les permiten afrontar con éxito esas situaciones problemáticas. 
Aunque muchos han opinado que afrontar los hechos negativos son habilidades personales e innatas, otros indican que ese desarrollo es propiciado por el ambiente familiar, la escuela y la sociedad. Tomando en cuenta que la resiliencia puede ser desarrollada en las familias y que puede producir el fortalecimiento de las capacidades de los individuos de afrontar con éxito los problemas, también ayudará a disminuir las situaciones de riesgo tales como el suicidio, el aborto, huir de la casa, entrar en relaciones no convenientes, etc.

\section{Características destacadas en las personas con resilien- cia}

El trabajo de Rimari Barrientos indica que las características de las personas que han desarrollado niveles adecuados de resiliencia son comúnmente sociales y competentes, indudablemente esta competencia se ha adquirido en algún lugar que generalmente es la familia. Habitualmente, ellos son poseedores de habilidades que le ayudan a enfrentar adecuadamente la vida como: el pensamiento crítico, la capacidad de resolver problemas y de tomar la iniciativa; además, son firmes en sus propósitos y poseen una visión positiva acerca de su futuro.

Cuando los niños ya hechos hombres enfrentan la vida, por lo general se caracterizan por ser capaces de establecer relaciones positivas, es decir los vínculos normales con los que se relacionan son de apoyo mutuo, de trabajo en equipo. Así resuelven problemas con facilidad, poseen un nivel elevado de automotivación para superar los problemas y hacer de ellos un trampolín al éxito.

\section{Dimensiones del modelo internacional de resilicien- cia de Saavedra}

Saavedra desarrolló, en el 2003, un modelo explicativo del comportamiento resiliente, el cual denominó "modelo internacional de resiliencia", a partir del cual la respuesta resiliente consistiría en una acción orientada a metas, una respuesta sustentada o vinculada a una visión amplia del problema y una adecuada visión de sí mismo. Ésta se caracteriza por elementos afectivos, cognitivos y proactivos ante los problemas, los cuales tienen como condición histórico-estructural un sistema de creencias y vínculos 
sociales que impregnan la memoria de seguridad básica. Ésta, de modo recursivo, interpreta la acción específica y los resultados.

Las dimensiones que se toman en cuenta para la resiliencia son: la ecuanimidad, perseverancia, confianza en sí mismo, satisfacción personal y sentirse bien solo.

La ecuanimidad denota una perspectiva balanceada de la propia vida y experiencias, es decir tomar las cosas tranquilamente y moderando sus actitudes ante la adversidad sin perder la calma.

La perseverancia indica la persistencia ante la adversidad o el desaliento, es decir, tener un fuerte deseo del logro y autodisciplina. En relación a la confianza en sí mismo, es la habilidad para creer en uno mismo, en sus capacidades y en sus posibilidades.

En relación a la satisfacción personal, comprende el significado de la vida y como se contribuye a ésta, es decir, el haber realizado algo bueno con el que uno se siente bien. Finalmente la dimensión de sentirse bien solo, tiene que ver con dar sig- nificado a la libertad, que somos únicos y muy importantes.

\section{Diseño metodológico}

El diseño de la investigación es no experimental y de corte transversal porque no se manipularon las variables, y porque los datos fueron tomados en un momento dado. El tipo de estudio fue correlacional.

\section{Población}

La población está conformada por 36 alumnos de género masculino matriculados en el 5to año de la Facultad de Teología de la Universidad Peruana Unión.

\section{Instrumentos de recolec- ción de datos}

El instrumento utilizado para medir la resiliencia es la Escala de Resiliencia por Wagnild y Young de 1993. Está compuesta de 25 ítems, los cuales puntúan en una escala tipo Likert de 7 puntos, donde 1 es en desacuerdo, y el máximo de acuerdo es 7. Los participantes indicaron el grado de conformidad con el ítem, ya que todos los ítems son calificados positivamente; los más altos puntajes serán in- 
dicadores de mayor resiliencia, el rango de puntaje varía entre 25 y 175 puntos. La Escala de Resiliencia tiene como componentes: confianza en sí mismo, ecuanimidad, perseverancia, satisfacción personal y sentirse bien solo.

Para la evaluación del funcionamiento familiar se aplicó el cuestionario de funcionamiento familiar (MMFF) elaborado por Atri y Zetune y el cual está basado en el modelo de funcionamiento familiar McMaster (McMaster of FamilyFunctioning MMFF). Este cuestionamiento es del tipo Likert y utiliza cinco opciones para valorar cada reactivo:

- Totalmente de acuerdo (5)

- Acuerdo (4)

- Ni de acuerdo ni en desacuerdo (3)

- En desacuerdo (2)

- Totalmente en desacuerdo (1)

Los factores que componen el cuestionario de Funcionamiento Familiar son: involucramiento afectivo, patrón de comunicación, resolución de problemas y control de conducta.

\section{Procedimiento para el aná- lisis de datos}

Los cuestionarios de funcionamiento familiar fueron calificados manualmente, de acuerdo a su tabla de puntuación y siguiendo el manual de calificación. Asimismo la Escala de Resiliencia, fue calificada en base a la escala de Likert de 7 puntos. Una vez obtenidos los datos sobre el funcionamiento familiar y resiliencia los resultados, fueron procesados a través del programa estadístico SPSS18 para Windows en Español. Las pruebas estadísticas en este estudio fueron las siguientes; a nivel descriptivo: tablas estadísticas y gráficos; y a nivel inferencial la Prueba de Kolmogorov-Smirnov para una muestra, para comprobar la normalidad y, posteriormente, la prueba de correlación de Pearson para comprobar la correlación entre las variables.

\section{Resultados y discusión}

Para responder la pregunta de investigación y determinar si existe una relación significativa entre la resiliencia y el funcionamiento familiar, primero se realizó una correlación entre funcionamiento familiar y resiliencia. 


\section{Tabla № 1 Correlación entre funcionamiento familiar y resiliencia}

\begin{tabular}{|l|l|l|l|}
\hline \multicolumn{4}{|c|}{ Correlaciones } \\
\hline \multirow{4}{*}{$\begin{array}{l}\text { Funcionamiento } \\
\text { Familiar }\end{array}$} & $\begin{array}{l}\text { Funcionamiento } \\
\text { Familiar }\end{array}$ & Resiliencia \\
\cline { 2 - 4 } & Correlación de Pearson & 1 &, $528(* *)$ \\
\cline { 2 - 4 } & Sig. (bilateral) & &, 001 \\
\cline { 2 - 4 } Resiliencia & $\mathrm{N}$ & 36 & 36 \\
\cline { 2 - 4 } & Correlación de Pearson &, $528(* *)$ & 1 \\
\cline { 2 - 4 } & Sig. (bilateral) &, 001 & 36 \\
\cline { 2 - 4 } & $\mathrm{N}$ & 36 & \\
\hline \multicolumn{2}{|c|}{$* *$ La correlación es significativa al nivel 0,01 (bilateral). } \\
\hline
\end{tabular}

Tabla 1. La correlación producto de Pearson indica que existe una relación moderada entre funcionamiento familiar y resiliencia $(R=0.528, p<0.001)$. Esto quiere decir que cuanto mayor es el nivel de funcionamiento familiar, mayor es la resiliencia. Los resultados obtenidos en esta investigación revelan que existe una moderada relación entre resiliencia y funcionamiento fa- miliar. Esto concuerda con la postura de Howdar, Druden y Jhonson quienes consideran que el ámbito familiar resulta muy importante para que se desarrolle la resiliencia, la familia influirá en la adquisición de habilidades que permitan sobrevivir y tener una conducta resiliente y adaptarse a situaciones de adversidad.

\section{Tabla No2 Correlación entre involucramiento afectivo y resiliencia}

\begin{tabular}{|c|c|c|c|}
\hline \multicolumn{4}{|c|}{ Correlaciones } \\
\hline & & Resiliencia & $\begin{array}{l}\text { Involucramiento } \\
\text { Afectivo }\end{array}$ \\
\hline \multirow{3}{*}{ Resiliencia } & Correlación de Pearson & 1 & $.451(* *)$ \\
\hline & Sig. (bilateral) & & .006 \\
\hline & $\mathrm{N}$ & 36 & 36 \\
\hline \multirow{3}{*}{$\begin{array}{l}\text { Involucramiento } \\
\text { Afectivo }\end{array}$} & Correlación de Pearson & $.451(* *)$ & 1 \\
\hline & Sig. (bilateral) & .006 & \\
\hline & $\mathrm{N}$ & 36 & 36 \\
\hline
\end{tabular}


Tabla 2. Podemos apreciar que existe relación significativa entre entre resiliencia y el involucramiento afectivo, esto nos da a entender que a mayor involucramiento afectivo mayor será la resiliencia de la persona. Los resultados obtenidos en esta investigación nos muestran que existe una relación moderada entre resiliencia e involucramiento afectivo. Raquel Atri y Zetune, menciona que el involucramiento afectivo es el grado en que la familia muestra interés y valora a cada miembro de la familia y denota la habilidad para responder con una amplia gama de emociones, así como la capacidad de proporcionar cuidados, afecto y bienestar y la investigación realizada nos muestra que éstas son algunas de las habilidades que una persona necesita para desarrollar resiliencia.

\section{Tabla No3 Correlación entre patrones de comunicación y resiliencia}

\begin{tabular}{|l|l|l|l|}
\hline \multicolumn{4}{|c|}{ Correlaciones } \\
\hline \multirow{4}{*}{ Resiliencia } & Resiliencia & $\begin{array}{l}\text { Patrones de } \\
\text { comunicación }\end{array}$ \\
\cline { 2 - 4 } & Correlación de Pearson & 1 & .129 \\
\cline { 2 - 4 } & Sig. (bilateral) & & .452 \\
\cline { 2 - 4 } & $\mathrm{N}$ & 36 & 36 \\
\hline \multirow{2}{*}{$\begin{array}{l}\text { Patrones de } \\
\text { comunicación }\end{array}$} & Correlación de Pearson & .129 & 1 \\
\cline { 2 - 4 } & Sig. (bilateral) & .452 & \\
\cline { 2 - 4 } & $\mathrm{N}$ & 36 & 36 \\
\hline \multicolumn{2}{|c|}{ ** La correlación es significativa al nivel 0,01 (bilateral). } \\
\hline
\end{tabular}

Tabla 3. La correlación producto de Pearson nos indica que no existe una relación significativa entre patrones de comunicación y resiliencia $(\mathrm{R}=0.129, \mathrm{p}<$ 0.452). Algunos investigadores indican que los patrones de comunicación tienen que ver con el intercambio de información verbal principalmente en el área instrumental. 
Tabla No4 Correlación entre solución de problemas y resiliencia

\begin{tabular}{|c|c|c|c|}
\hline \multicolumn{4}{|c|}{ Correlación } \\
\hline & & Resiliencia & $\begin{array}{l}\text { Solución de } \\
\text { Problemas }\end{array}$ \\
\hline \multirow[t]{3}{*}{ Resiliencia } & Correlación de Pearson & 1 & $.617(* *)$ \\
\hline & Sig. (bilateral) & & .000 \\
\hline & $\mathrm{N}$ & 36 & 36 \\
\hline \multirow[t]{3}{*}{ Solución de Problemas } & Correlación de Pearson & $.617(* *)$ & 1 \\
\hline & Sig. (bilateral) & .000 & \\
\hline & $\mathrm{N}$ & 36 & 36 \\
\hline
\end{tabular}

Tabla 4. Como se puede apreciar en la correlación producto de Pearson, nos indica que existe una relación moderada entre solución de problemas y resiliencia $(\mathrm{R}=0.617, \mathrm{p}<0.000)$. Los investigadores de la resiliencia concuerdan en que la solución de problemas se da cuando la familia es capaz de ponerse de

acuerdo y que tiene una buena organización familiar; por lo tanto, se infiere que puedan resolver los problemas instrumentales que se les presentan. En la investigación realizada se encontró que para decir que una persona es resiliente, es necesario que tenga la capacidad de resolver problemas.

\section{Tabla No5 Correlación entre patrones de conducta y resiliencia}

\begin{tabular}{|l|l|l|l|}
\hline Correlación & & Resiliencia & $\begin{array}{l}\text { Patrones de } \\
\text { conducta }\end{array}$ \\
\hline \multirow{3}{*}{ Resiliencia } & Correlación de Pearson & 1 & .316 \\
\cline { 2 - 4 } & Sig. (bilateral) & & .060 \\
\cline { 2 - 4 } & $\mathrm{N}$ & 36 & 36 \\
\hline Patrones de conducta & Correlación de Pearson & .316 & 1 \\
\cline { 2 - 4 } & Sig. (bilateral) & .060 & \\
\cline { 2 - 4 } & $\mathrm{N}$ & 36 & 36 \\
\hline \multirow{3}{*}{$* *$ La correlación es significativa al nivel 0,01 (bilateral). } \\
\hline
\end{tabular}


Tabla 5. Como se puede apreciar, en la correlación producto de Pearson, nos indica que no existe una relación significativa entre patrones de conducta y re- siliencia $(R=0.316, p<0.060)$. Esto quiere decir que cuanto mayor es el nivel de funcionamiento familiar mayor es la resiliencia.

\section{Tabla No 6 Tabla de contingencia}

\begin{tabular}{|l|l|l|l|l|l|}
\hline $\begin{array}{l}\text { Funcionamiento } \\
\text { familiar }\end{array}$ & & $\begin{array}{l}\text { Resiliencia } \\
\text { Baja }\end{array}$ & $\begin{array}{l}\text { Resiliencia } \\
\text { Promedio }\end{array}$ & $\begin{array}{l}\text { Resiliencia } \\
\text { Alta }\end{array}$ & Total \\
\hline \multirow{2}{*}{ Disfunción } & Recuento & 1 & 2 & 0 & 3 \\
\cline { 2 - 6 } & $\%$ del total & $2,8 \%$ & $5,6 \%$ &, $0 \%$ & $8,3 \%$ \\
\hline \multirow{2}{*}{$\begin{array}{l}\text { Medianamente } \\
\text { Funcional }\end{array}$} & Recuento & 3 & 7 & 0 & 10 \\
\cline { 2 - 6 } Funcional & $\%$ del total & $8,3 \%$ & $19,4 \%$ &, $0 \%$ & $27,8 \%$ \\
\hline & Recuento & 2 & 9 & 12 & 23 \\
\cline { 2 - 6 } & $\%$ del total & $5,6 \%$ & $25,0 \%$ & $33,3 \%$ & $63,9 \%$ \\
\hline Total & Recuento & 6 & 18 & 12 & 36 \\
\hline
\end{tabular}

Tabla 6. En ella se aprecia que $33.3 \%$ de los alumnos del 5 to año de la Facultad de Teología de la Universidad Peruana Unión, califican a sus familias como más funcionales y presentan niveles altos de resiliencia; un $19.4 \%$ de los alumnos califican a sus familias como medianamente funcionales $y$ presentan resiliencia promedio y un $2.8 \%$ se caracteriza por calificar a sus familias como disfuncionales y presentar niveles bajos de resiliencia. Esta tabla corrobora lo que la mayoría indica referente a que el papel de la familia va mucho más allá de asegurar la supervi- vencia física de sus miembros, ya que garantiza la integración sociocultural de éstos a los escenarios donde les toca vivir como personas.

También se señala que, el buen funcionamiento de la familia, al satisfacer las necesidades básicas materiales y psicológicas, asegura el desarrollo de personalidades adultas estables, integradas, maduras emocionalmente, haciendo posible un funcionamiento interpersonal constructivo, al proporcionar un medio humano apropiado, durable, consistente, íntimo y asegurador. 


\section{Conclusiones}

De acuerdo a los resultados presentados en esta investigación sobre la relación entre funcionamiento familiar y resiliencia de los alumnos del 5to año de la Facultad de Teología de la Universidad Peruana Unión, se llegó a las siguientes conclusiones:

En relación a la hipótesis de investigación, se encontró una correlación moderada entre el funcionamiento familiar y la resiliencia, pues según la correlación de producto de Pearson indica una $\mathrm{R}=0.528, \mathrm{p}<0.001$.

Con respecto al objetivo general, se encuentra que el funcionamiento familiar se relaciona significativamente con el nivel de resiliencia de los alumnos de 5to año de la Facultad de Teología. Estos estudiantes con nivel alto de resiliencia califican como más funcionales en la mayoría de las dimensiones del cuestionario del funcionamiento familiar, excepto en el funcionamiento de patrones de comunicación.

Con respecto a nuestro primer objetivo específico, se encontró que la dimensión de
Involucramiento afectivo, sí se relaciona significativamente con el nivel de resiliencia de los alumnos de 5to año de la Facultad de Teología $(\mathrm{R}=0.451, \mathrm{P}=0.006)$.

Con respecto al segundo objetivo específico, se encontró que la dimensión de Patrones de Comunicación, no se relaciona significativamente con el nivel de resiliencia de los alumnos de quinto año de la Facultad de Teología $(\mathrm{R}=0.129$, $\mathrm{P}=0.452$ ).

Referente al tercer objetivo específico, se encontró que la dimensión de solución de problemas, se relaciona significativamente con el nivel de resiliencia de los alumnos de 5to año de la Facultad de Teología $(\mathrm{R}=0.617$, $\mathrm{P}=0.000$ ).

Con respecto al cuarto objetivo específico, se encontró que la dimensión de Patrones de conducta, no se relaciona moderadamente con el nivel de resiliencia de los alumnos de 5to año de la Facultad de Teología $(\mathrm{R}=0.316, \mathrm{P}=0.060)$.

\section{Recomendaciones}

Se recomienda tomar en cuenta la realización de una evaluación 
de funcionamiento familiar en los alumnos de las diferentes facultades de la Universidad Peruana Unión.

Establecer programas de apoyo con el fin de elevar el nivel de resiliencia de los alumnos universitarios de la Universidad Peruana Unión.

Se recomienda realizar estudios adicionales que permitan conocer el funcionamiento familiar y la resiliencia en familias adventistas.

A partir del presente estudio, se recomienda realizar investigaciones futuras que relacionen la resiliencia y la vida práctica religiosa de los alumnos de la Facultad de Teología.

Realizar el tema de investigación en otras poblaciones de la misma universidad para ver sus diferencias.

\section{Bibliografía}

Atri y Zetune, Raquel. Confiabilidad y validez del cuestionario de evaluación del funcionamiento familiar EFF. México: Tesis de Maestría Universidad de las Américas, 1987.
Atri y Zetune, R. "Cuestionario de evaluación del funcionamiento familiar (EFF)". En Velasco, M.L. y Luna, M. Comps. Instrumentos de evaluación en terapia familiar y de pareja. México: Pax, 2006.

Howard S., Dryden, J. y Johnson B., Chilhood Resilience: Review and Critique of Literature. Oxford Review of Education, 1999.

Nardone, G.Giannotti, E. y Rocchi, R., Modelos de familia: conocer y resolver los problemas entre padres e hijos. Herder. Barcelona, 2003

Rimari Barrientos, Luis Enrique. "La resiliencia ¿cómo forjar personas resilientes en nuestra sociedad?", Revista pedagógica del maestro peruano. Lima: Asociación cultural San Jerónimo, 2008. 